\title{
Preliminary Qualitative Evaluation of Patient-Related Perspectives Related to the Implementation of a Predictive Algorithm in a Telehealth System for COPD
}

\author{
Clara BENDER $^{\mathrm{a}, 1}$, Stine HANGAARD ${ }^{\mathrm{a}, \mathrm{b}}$, Thomas KRONBORG ${ }^{\mathrm{a}, \mathrm{b}}$, \\ Ole Kristian HEJLESEN ${ }^{\mathrm{a}}$ and Pernille Heyckendorff SECHER ${ }^{\mathrm{a}}$ \\ ${ }^{a}$ Department of Health Science and Technology, Aalborg University, Fredrik Bajers \\ Vej 7C, 9220 Aalborg East, Denmark \\ b Steno Diabetes Center, North Denmark, Søndre Skovvej 3E, 9000 Aalborg, Denmark
}

\begin{abstract}
The aim of the present study was to evaluate patient-related perspectives from a five-week test of the implementation of a COPD prediction algorithm. The test intended to discover and avoid potential errors prior to testing the COPD prediction algorithm in a two-armed randomized controlled trial (RCT). The COPD prediction algorithm aims to predict exacerbations in COPD based on home measurements. In the present study, the algorithm was implemented in a currently deployed telehealth system. Five weeks after implementation, six interviews were conducted, including five interviews with patients with COPD and one interview with a specialized COPD nurse. The participants were overall satisfied with the telehealth system including the COPD prediction algorithm. However, technical issues must be addressed before the COPD prediction algorithm is ready to be tested in the RCT. Moreover, communication with the monitoring nurses should be clearer based on the COPD nurse's experiences. In conclusion, the participants were satisfied with the integration of the COPD prediction algorithm in the telehealth system. The identification of technical issues shows the importance of including a technical test period in a similar trial setup.
\end{abstract}

Keywords. COPD, telemedicine, predictive analytics, RCT, interview, qualitative research

\section{Introduction}

The number of people suffering from chronic obstructive pulmonary disease (COPD) is increasing and COPD is now the third leading cause of morbidity and mortality worldwide (1). The mortality rate is expected to exceed to more than 4.5 million deaths annually by 2030 (2). Substantial economic costs are related to COPD treatments etc. (3). Suffering from COPD affect the individual person both physically and mentally. Several studies show that patients with COPD have reduced quality of life $(3,4)$. This reduction increases with the number of exacerbations since the frequency of exacerbations contribute to the worsening of COPD at a long-term level (5). Early treatment of exacerbations

\footnotetext{
${ }^{1}$ Corresponding Author, Clara Bender, The Department of Health Science and Technology, Aalborg University, Fredrik Bajers Vej 7 E1-204, 9220, Aalborg East, Denmark; E-mail: csch@hst.aau.dk
} 
is associated with faster recovery and decreased risk of emergency hospitalizations (6). Therefore, it is important to predict exacerbations in order to facilitate early and preventive treatment $(6,7)$. Several telehealth studies have attempted to prevent exacerbation with varying effects $(6,8)$. Thus, telehealth intervention in COPD should be optimized.

Telehealth interventions in COPD may be optimized by applying predictive algorithms. Until now a predictive algorithm in COPD with clinically useful quality has not been developed (9). However, Kronborg et al. have developed a COPD prediction algorithm based on home measurements of physiological parameters (blood pressure, oxygen saturation and pulse), that manages to predict exacerbations in patients with COPD (10). The COPD prediction algorithm by Kronborg et al. seeks to support clinical decisions among specialized COPD nurses by predicting COPD exacerbations prior to onset. Hence, the hypothesis is that the COPD prediction algorithm can reduce the number of acute hospitalizations and improve the health-related quality of life among patients with COPD. The COPD prediction algorithm will be tested in a two-armed RCT with a sixmonth study period. Patients with COPD, who already receive telehealth as part of their treatment, will be included in the trial. Before running such a trial set-up, it is important to record potential pitfalls regarding patient-related related to the implementation of the COPD prediction algorithm.

The aim of the present study is to evaluate patient-related perspectives from a fiveweek test period in order to discover and avoid potential errors before large-scale testing of the COPD prediction algorithm.

\section{Methods and Materials}

\subsection{Study design}

The study was a preliminary qualitative evaluation of a COPD prediction algorithm. Selected participants with COPD used a telehealth system including the COPD prediction algorithm for five weeks. The participants were using the telehealth system prior to the study, but during the study, they were instructed to measure their physiological parameters three times a week, which is more often than usual. These additional measurements were prompted to the patients through a digital questionnaire built into the telehealth system. The algorithm was tested by exploring patient-related perspectives through qualitative interviews by the end of the study.

The included partners in the test period were Aalborg University and partners from the North Denmark Region. Interviews were conducted with a specialized COPD nurse and with the participants with COPD. The partners from the North Denmark Region' role was to make sure that ongoing updates of the software was available to the COPD participants.

\subsection{Participants}

Five participants (three women and two men) with COPD were recruited from the district Health Center Aalborg in Aalborg Municipality, Denmark. In addition, a specialized COPD nurse employed at the same care district was chosen to identify the participants. The nurse in question knew the participants beforehand as she was monitoring their data. In advance, the specialized COPD nurse knew the participants' ability to try something new, the status of their condition, and their motivation. Among others, her role was to 
describe her experiences with the system and test whether the algorithm worked as well as to ensure that she felt prepared to the upcoming RCT. The five participants were all familiar with telehealth and were able to cooperate in a test period; moreover, the participants should be able to and willing to measure their oxygen saturation and heart rate three times per week and measure their blood pressure once a week.

\subsection{Data collection method}

Prior to conducting the semi-structured interviews, two interview guides were prepared. One interview guide was prepared for the interview with the specialized COPD nurse and one was prepared for the individual interviews with the participants with COPD. Both interview guides consisted of three themes: 1) About the participant or COPD nurse, 2) The design of the system and 3) Utility of the trial.

The interviews were conducted by telephone to avoid physical contact due to the COVID-19 pandemic. The duration of the interviews lasted between 5-15 minutes with the COPD participants and 30 minutes with the specialized COPD nurse. To remember relevant points from the interviews, each telephone interview was recorded by Dictaphone. Afterwards, the researchers coded and identified relevant themes by using a qualitative thematic analysis based on a hermeneutic phenomenological approach.

\section{Results}

Table 1 presents the demographic information of the participants with COPD included in the study. The average age of the participants was 65.2 years and the average number of years diagnosed with COPD was 11.6 years. All participants were retired or unable to work and most of them had comorbidities.

Table 1. Demographic information of the participants with COPD.

\begin{tabular}{|c|c|c|c|c|c|}
\hline Participant no. & $\# 1$ & $\# 2$ & $\# 3$ & $\# 4$ & $\# 5$ \\
\hline Sex & Woman & Woman & Woman & Male & Male \\
\hline Age & 65 & 65 & 62 & 65 & 69 \\
\hline Postal code & 9200 & 9370 & 9200 & 9000 & 9220 \\
\hline Civil status & Single & Married & Single & Married & Married \\
\hline Employment & No job & No job & No job & No job & No job \\
\hline Education level & Craftsman & State school & State school & Master's degree & Craftsman \\
\hline $\begin{array}{c}\text { Years with diag- } \\
\text { nosed COPD }\end{array}$ & 10 & 5 & 3 & 10 & 30 \\
\hline $\begin{array}{r}\text { Smoked last } 6 \\
\text { months? }\end{array}$ & No & No & Yes & No & No \\
\hline Other diseases & No & $\begin{array}{r}\text { Crohn's dis- } \\
\text { ease }\end{array}$ & Osteoporosis & $\begin{array}{r}\text { Cardiovascular } \\
\text { disease }\end{array}$ & Cancer \\
\hline
\end{tabular}

Three themes were emerged from the interviews with the five participants: 1) Experiences of using a telehealth system when living with COPD, 2) Extra measurements increased safety, control and disease understanding, and 3) The development of new functions in a telehealth system led to technical issues. Two themes were emerged from the interview with the COPD nurse: 1) Understanding the nurse's role and work functions related to the upcoming RCT, and 2) Clarification of new system functionalities in the telehealth system.

Table 2 gives an insight into the distribution of responses linked to selected questions and discovered themes from the five interviews with the participants. As it appears in 
table 2, question no. 4, 5, 6 and 7 included patient-related aspects of the COPD prediction algorithm.

Table 2. A recapitulation of the answers from the questions asked in the five interviews.

\begin{tabular}{|c|c|c|}
\hline No. & Question & Number of distribution of answers \\
\hline & $\begin{array}{c}\text { What is your experience with } \\
\text { technology in general }\end{array}$ & $\begin{array}{l}4 \text { out of } 5 \text { have experience with technology, } 1 \text { does } \\
\text { not answer the question }\end{array}$ \\
\hline & $\begin{array}{c}\text { What was your experience of the } \\
\text { new questionnaire? }\end{array}$ & $\begin{array}{l}3 \text { out of } 5 \text { said it was fine to use the questionnaire, } 2 \\
\text { out of } 5 \text { have not had the questionnaire available }\end{array}$ \\
\hline & $\begin{array}{l}\text { How did the updates affect you } \\
\text { during the test period? }\end{array}$ & $\begin{array}{c}\text { Various responses, in general there were problems } \\
\text { with the updates }\end{array}$ \\
\hline & $\begin{array}{l}\text { Do you feel any benefits of the } \\
\text { extra measurements? }\end{array}$ & $\begin{array}{l}3 \text { out of } 5 \text { experienced increased control, } 2 \text { out of } 5 \\
\text { experienced increased safety, and } 2 \text { out of } 5 \text { experi- } \\
\text { enced increased understanding of their disease }\end{array}$ \\
\hline
\end{tabular}

Below, selected quotations from the interviews with the COPD participants and the specialized COPD nurse are given.

In general, the participants found it easy to use the telehealth system and all of them had prior experience with technology. One participant answered: "I used technology when I was working, so it is easy for me". The new questionnaire for the additional measurements was not always available for the participants: "I did not have the questionnaire available in the first week". Three out of five answered the same. Some participants did not turn off their telehealth system and therefore they did not receive the updated version of the questionnaire. One participant answered: "it gave some problems in the beginning". On the contrary, another participant answered that she did not experience any problems after the update. The general attitude towards the extra measurements was that it increased the participants' feeling of control, safety and understanding of their disease: "I feel much more safe, secure and satisfied when measuring extra oxygen saturations". All the participants were motivated to continue to perform the extra oxygen saturation measurements. None of the participants felt it was a burden during the five-week test period.

From the interview with the specialized COPD nurse it appeared that, the nurse was looking for a flag item in the telehealth system when an exacerbation occurred and not an alarm bell. The nurse highlighted that the telehealth system with the COPD prediction algorithm was not an alarm system with an acute line, but an extra service offer for patients with COPD.

\section{Discussion}

The present study aimed to evaluate patient-related perspectives from a test period after implementation of a COPD prediction algorithm in a telehealth system. In general, the participants were positive towards the telehealth system that included a COPD prediction algorithm. However, minor technical issues were reported due to the implementation of the algorithm.

The participants in general were used to apply technology in their daily life. This information is relevant, since the upcoming RCT is based on the assumption that the participants are able to use technology to measure their physiological parameters. The results also indicate the importance of ensuring that the participants always have the latest updated version of the telehealth system. This highlights the importance of iterative steps where errors are removed $(12,13)$. However, very often when RCTs and studies developing and testing algorithms are planned, the test period, where potential clinical 
problems can be identified, is skipped. This might be due to the interests of the algorithm developers who have less focus on the softer aspects of the system such as patient-related perspectives (12). This study might be the first that actually conducted a test period that focus on the softer patient-related perspectives and aspects of testing a COPD prediction algorithm into an already running telehealth system. Potential miscommunication regarding a flag vs. bell alarm item can now be avoided, since the monitoring specialized COPD nurses in the RCT, will receive more clear information regarding new symbols in the telehealth system and the interpretation hereof. Again, this highlights the importance of iterative steps within a system developing circle $(12,13)$.

In conclusion, the participants with COPD were satisfied with the inclusion of the prediction algorithm in a telehealth system. Likewise, the specialized COPD nurse felt prepared to monitor after participating in the test period. However, all relevant questionnaires must be available for participants in the system and the identified technical issues must be corrected before the RCT. Future work includes testing the developed COPD prediction algorithm in the upcoming RCT.

\section{References}

[1]. Iheanacho I, Zhang S, King D, Rizzo M, Ismaila AS. Economic burden of chronic obstructive pulmonary disease (COPD): A systematic literature review. Int J COPD 2020;15:439-60. https://doi.org/10.2147/COPD.S234942

[2] Organization WH. Projections of mortality and causes of death, 2015 and 20302019

[3] Anzueto A. Impact of exacerbations on copd. Eur Respir Rev 2010;19:113-8. https://doi.org/10.1183/09059180.00002610

[4] Halpin DMG, Miravitlles M, Metzdorf N, Celli B. Impact and prevention of severe exacerbations of COPD: A review of the evidence. Int J COPD 2017;12:2891-908. https://doi.org/10.2147/COPD.S139470

[5] Donaldson GC, Law M, Kowlessar B, Singh R, Brill SE, Allinson JP, et al. Impact of prolonged exacerbation recovery in chronic obstructive pulmonary disease. Am J Respir Crit Care Med 2015;192:943-50. https://doi.org/10.1164/rccm.201412-2269OC

[6] Wilkinson TMA, Donaldson GC, Hurst JR, Seemungal TAR, Wedzicha JA. Early Therapy Improves Outcomes of Exacerbations of Chronic Obstructive Pulmonary Disease. Am J Respir Crit Care Med 2004;169:1298-303. https://doi.org/10.1164/rccm.200310-1443OC

[7] Tomasic I, Tomasic N, Trobec R, Krpan M, Kelava T. Continuous remote monitoring of COPD patientsjustification and explanation of the requirements and a survey of the available technologies. Med Biol Eng Comput 2018;56:547-69. https://doi.org/10.1007/s11517-018-1798-z

[8] Quaderi SA, Hurst JR. The unmet global burden of COPD. Glob Heal Epidemiol Genomics 2018;3:9-11. https://doi.org/10.1017/gheg.2018.1

[9] Sanchez-Morillo D, Fernandez-Granero MA, Leon-Jimenez A. Use of predictive algorithms in-home monitoring of chronic obstructive pulmonary disease and asthma: A systematic review. Chron Respir Dis 2016;13:264-83. https://doi.org/10.1177/1479972316642365

[10] Kronborg T, Hangaard S, Cichosz SL, Hejlesen OK. A two-layer probabilistic model to predict COPD exacerbations for patients in telehealth. Comput Biol Med 2020

[11] Udsen F, Lilholt P, Hejlesen O, Ehlers L. Effectiveness and cost-effectiveness of telehealthcare for chronic obstructive pulmonary disease: study protocol for a cluster randomized controlled trial. Trials 2014;15:178. https://doi.org/10.1186/1745-6215-15-178

[12] Hartson HR, Andre TS, Williges RC. Criteria for evaluating usability evaluation methods. Int J Hum Comput Interact 2003;15:145-81. https://doi.org/10.1207/S15327590IJHC1501_13

[13] Lilholt PH, Schaarup C, Hejlesen OK. An Iterative, Mixed Usability Approach Applied to the Telekit System from the Danish TeleCare North Trial. Int J Telemed Appl 2016;2016. https://doi.org/10.1155/2016/6351734 